\title{
VLBI PHASE-REFERENCING FOR OBSERVATIONS OF WEAK RADIO SOURCES
}

\section{J.-F LESTRADE}

\author{
Observatoire de Meudon, DERAD \\ F-92195, Meudon, Principal Cedex \\ and \\ Jet Propulsion Laboratory, California Institute of Technology, \\ 4800 Oak Grove Drive, Pasadena, California, 91011
}

\begin{abstract}
Phase-referencing is a technique used in VLBI to extend the signal coherence time from a few minutes to a few hours in order to enhance significantly its sensitivity. With this technique, VLBI observations of milliJansky radio sources can be conducted for high-accuracy differential astrometry as well as imaging. We describe the technique in some details and present, as an example, a submilliarcsecond differential astrometric experiment design to identify the star responsible for the weak radio emission in the binary system Algol.
\end{abstract}

\section{INTRODUCTION}

Very Long Baseline Interferometry (VLBI) is used very successfully to map the milliarcsecond structure of radio sources and to build the most accurate celestial and terrestrial reference frames to monitor the orientation of the Earth crust relative to sideral space. For mapping radio sources, techniques based on closure phases, like hybrid mapping, are used. For global astrometry and geodesy, the prime observable is the group delay measured by the Band Width Synthesis Technique. These VLBI techniques have been applied almost exclusively on strong radio extragalactic sources, i.e. sources with flux densities $\geq 100 \mathrm{mJy}$ with the Mark III data acquisition system (Rogers et al 1983). However, a variety of weaker radio sources, like radio emitting stars, super nova remanants, pulsars..., also requires VLBI studies.

Sensitivity of VLBI is limited 1) by the high detection threshold (SNR $\geq 7$ ) required for real VLBI fringes and 2) by the short signal coherence time to reach this SNR. The high detection threshold in VLBI is due to the fringe search that must be carried out over a relatively large domain of the delay and delay-rate space to find the offset and drift between the station clocks which are unknown $a$ priori. The short coherence time in VLBI is due to high-order phase instabilities of the atmosphere or of the station clocks which cannot be modelled by the linear function representing the clock and drift of the fringe search.

In Figure 1, the continuous thick line is the Allan variance function plotted with experimental data acquired with an hydrogen maser frequency and time standard typically used at VLBI stations. The dotted line shows the Allan variance of VLBI delay-rate residuals of actual data at $89 \mathrm{GHz}$ (Rogers et al 1984) and complemented with the statistical model of the wet troposphere by Treuhaft 
and Lanyi (1987) for durations longer than 800 seconds. This figure indicates that rapid fluctuations of the wet component of the troposphere are mainly responsible for coherence loss for VLBI data integration times smaller than $10^{4}$ sec while station maser clocks instabilities dominate beyond. In practice, for frequencies of observation in the $\mathrm{GHz}$ range, integration times $T \leq 10^{3} \mathrm{sec}$ are chosen to make coherence loss negligeable in VLBI.

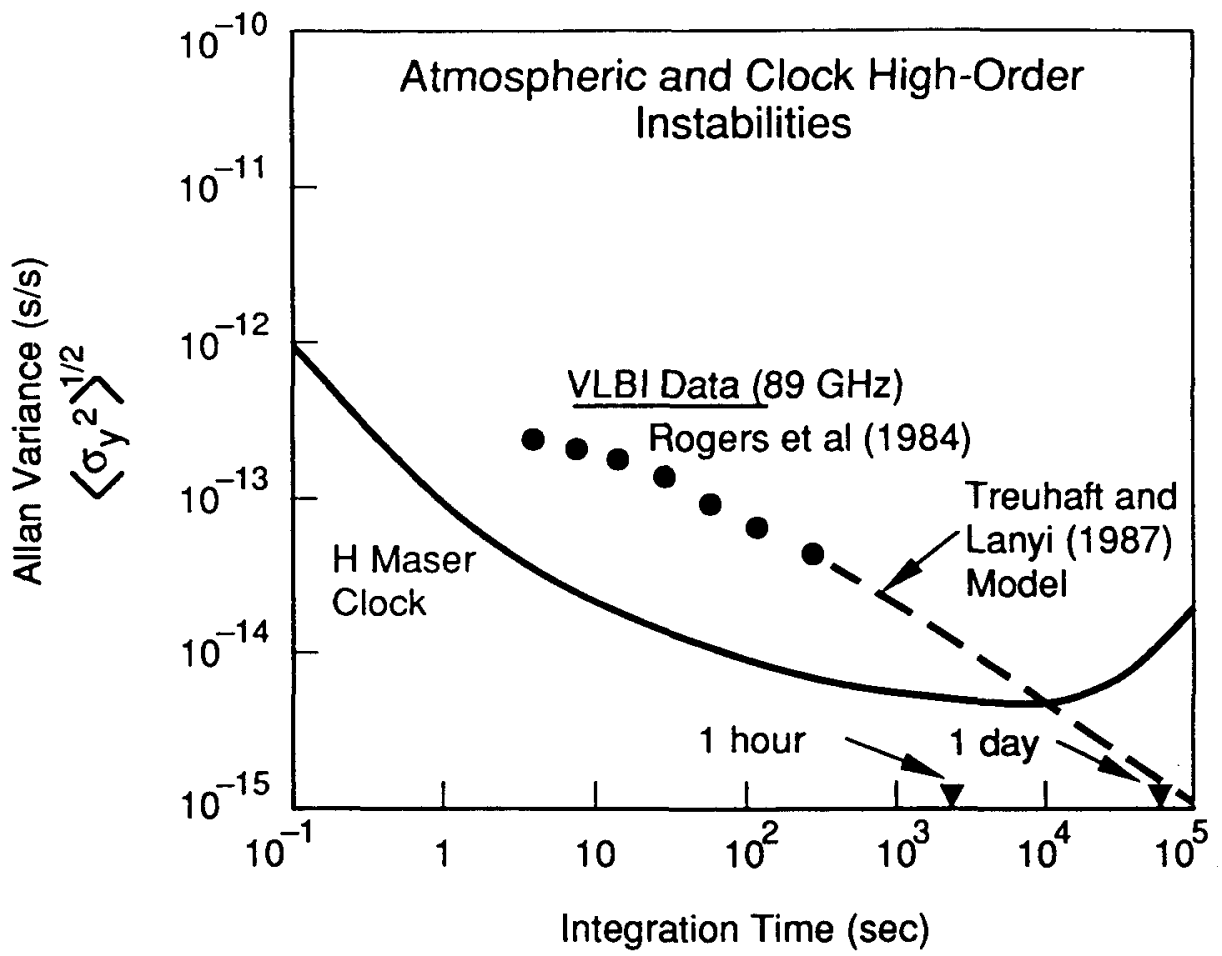

Fig. 1 : Allan Variance functions showing the fractional frequency instabilities of a VLBI station clock and of the wet component of the troposphere

\section{ENHANCEMENT OF VLBI SENSITIVITY}

The proper strategy of observations can yield a substantial increase of the signal coherence time $T$ to enhance VLBI sensitivity $(\operatorname{SNR} \propto \sqrt{T})$.

a) Observation strategy :

A reference for the VLBI phase can be established throughout an entire experiment by observing a strong source angularly close to the weak program source. If the position and structure of the strong reference source is accurately known, the variations of its residual phase monitor the atmospheric and instrumental fluctuations. Hence, these effects can be removed from the weak program source phase and the weak signal coherence time extended to several hours. Since the reference and program sources are rarely angularly close enough to be in the antenna beam, observations must be conducted alternately on the two sources 
with a switching cycle (program source observation + antenna slew + reference source observation) which is a few minutes as limited by antenna technology.

b) Correlator strategy :

This switching cycle of a few minutes limits the integration time for each individual scan to 1-2 minutes. This is usually too short for reliable VLBI detection of the weak program source fringes by the classical search over a large domain of delay and delay-rate space. Instead, delay and delay-rate tracking of the weak source fringes is possible by using the residual delay and delay-rate of the strong reference source to add coherently the weak instantaneous visibilities. Instantaneous visibilities are incoherent averages of the recorded VLBI data produced by the correlator over very short accumulation periods of $\sim 1 \mathrm{sec}$.

The basic idea of this tracking (Fig. 2) is that the a priori unknown station clock offset and drift contribute identically to the delay and delay-rate residuals of the reference and program suurces if their a priori angular separation is accurate enough to produce biaises in the correlator model which are not larger than a small fraction of the delay beam $\frac{1}{B}$ ( $B$ is the spanned bandwidth) and of the delay-rate beam $\frac{1}{\Delta T}$ ( $\Delta T$ is the integration time of each individual observation).

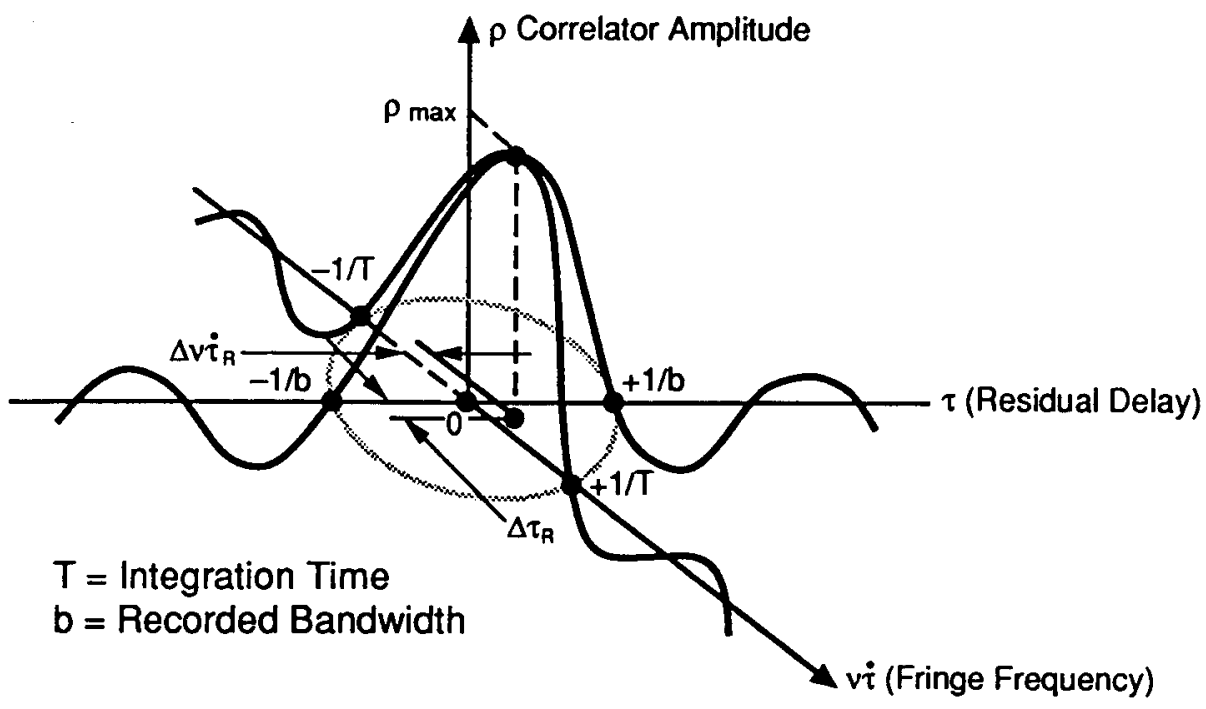

Fig. 2 : Concept of the delay and delay-rate tracking to find weak VLBI fringes with the strong reference source residuals $\left(\Delta \tau_{R}, \Delta \nu \dot{\tau}_{R}\right)$.

Practically, for a $3500 \mathrm{~km}$ continental baseline observing at $5 \mathrm{GHz}$, for $B=28 \mathrm{MHz}$ and $\Delta T=60 \mathrm{sec}$, the a priori source separation must be known to $\sim 0.1^{\prime \prime}$ to locate the weak VLBI fringes within a $\sim 1 / 6$ of the delay - delay rate beam. 
c) Differenced visibilities :

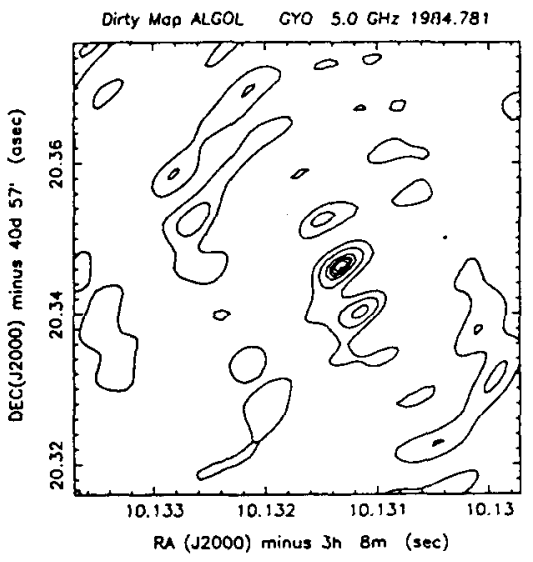

Dirty Beem ALGOL GYO $5.0 \mathrm{GHz} 1984.781$

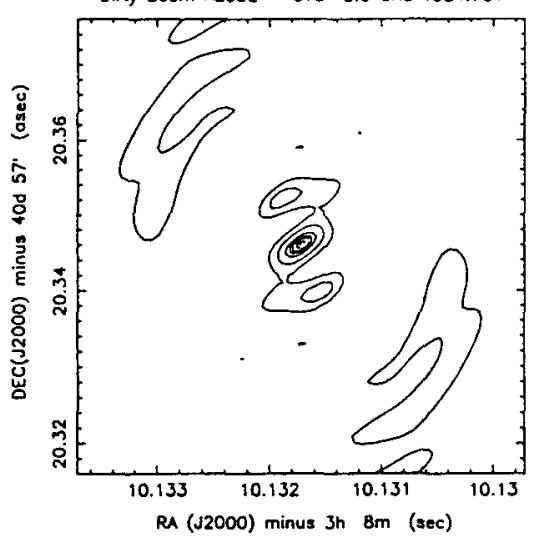

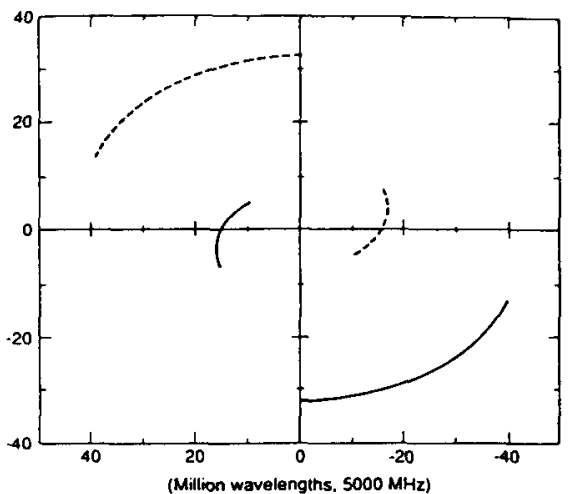

FIG. $\downarrow . u-v$ coverage cor responding to the phase-referenced diny map of Algoi.
FiG. 3. Dirty map of Algol (peak $=3 \mathrm{~mJ} y$ ) produced by Fourier inversion of the visibilities from both circular polarizations on the two baselines OVRO-VLA (OY) and Greenbank-VLA (GY). The measured phases of Algol were calibrated with the extragalaciic source $0309+411$ used as a phase-reference source. This map provides the precise position of Algol relative $100309+411$. The ditty beam is also shown. Contour levels are $20,90,75,85,90,95$. and $99 \%$ of the peak.

The visibilities measured on the reference and program sources with the strategy described above can be used for high-accuracy differential astrometry and for mapping. For these applications, the crude correlator model phases must be restored to form residual phases anew with a more accurate astrometric model. A complete model is described in Sovers and Fanselow (1987). Then, each new residual phase of the reference source must be extrapolated over a few minutes to the nearest time $t_{k}$ of observation of the program source unless simultaneous observations were conducted. Finally, at all the times $t_{k}$, the resulting residual visibilities of the reference aild program sources are differenced and are finally Fourier inverted:

$$
\Gamma(\alpha, \delta)=\sum_{\alpha} \sum_{\delta} \sum_{k=1}^{N} \rho_{k} \times \exp \left(-i\left[\phi^{W}\left(\alpha, \delta, t_{k}\right)-\phi^{R}\left(t_{k}\right)\right]\right)
$$


$\rho_{k}=$ amplitude of the visibility of the program source measured at $t_{k}$.

$\phi^{W}\left(\alpha, \delta, t_{k}\right)=$ residual phase of the visibility of the weak program source measured at time $t_{k} . \alpha$ and $\delta$ are the sky coordinates of the program source brightness distribution around the a priori source position.

$\phi^{R}\left(t_{k}\right)=$ residual phase of the visibility of the strong reference source extrapolated at time $t_{k}$.

The basic idea of VLBI phase-referenced observations is that the unknown atmospheric and station clock phase instabilities vanish in the phase difference $\phi^{W}\left(\alpha, \delta, t_{k}\right)-\phi^{R}\left(t_{k}\right)$ of equation (1) to a high degree if the source separation is small.

Figure 3 above shows an example of the result of such data processing presented in details in Lestrade et al (1990). The dirty map, beam and u-v coverage of this figure are from an experiment on the radio star Algol at $5 \mathrm{GHz}$ when its flux density was as low as $3 \mathrm{mJy}$ on 1984 October 11 . The peak in the dirty map corresponds to an SNR of 12 for a total integration time of 4.2 hours with no significant coherence loss due to systematic phase biaises. This was achieved by alternately observing Algol and $0309+411$, separated by $1^{\circ}$, with a 3-station array formed by the phased-VLA, Greenbank and Owens Valley and with the Mark III data acquisition system (recorded band width $B=28 \mathrm{MHz}$ ). The Haystack Mark III correlator and software were used to carry out the delay and delay-rate tracking of the weak program source VLBI fringes. The Software for Phase-Referenced INTerferometry (SPRINT) was used to form new accurate residual phases and to coherently add the differenced visibilities of the entire experiment with equation (1).

d) Accuracy of the astrometric model for $\phi^{W}\left(\alpha, \delta, t_{k}\right)$ and $\phi^{R}\left(t_{k}\right)$ of eq (1):

Systematic errors in the astrometric model induce systematic errors in the differenced residual phase $\Delta \phi\left(t_{k}\right)=\phi^{W}\left(\alpha, \delta, t_{k}\right)-\phi^{R}\left(t_{k}\right)$ of equation (1) which are scaled down with the separation in radian between the reference and program sources (e.g. reduction factor 57 for a $1^{\circ}$ separation). These systematic errors in $\Delta \phi\left(t_{k}\right)$ must be a small fraction of the observing wavelength to use equation (1) for high-accuracy differential astrometry and for mapping the structure of the weak program source.

It is usual to model the phase measured over VLBI baseline by splitting into geometry, propagation and structure effects. The geometric part $\phi=f \times \frac{\vec{b} \cdot \vec{\sigma}}{c}$ ( $f$ : observing frequency, $\vec{b}$ : baseline vector, $\vec{\sigma}:$ unit-vector of source direction) is the most accurately modelled, i.e. systematic error in the difference $\Delta \phi=f \times \frac{\vec{b} \cdot \vec{\sigma}}{c} \times \Delta \theta$ can be less than $10^{\circ}$ for a source separation $\Delta \theta=1^{\circ}$ at $f=5 \mathrm{GHz}$ on an intercontinental baseline $b=8000 \mathrm{~km}$.

Propagation effects due to the troposphere and the ionosphere are spatially and temporally variable and are the most difficult to model accurately. The total zenith phase-delay contributions of the troposphere are $\sim 200 \mathrm{~cm}$ for the dry component and $\sim 20 \mathrm{~cm}$ for wet component and can be modelled with external measurements (e.g. meteorological data and radio water wapor radiometer) and computed along the line of sight with a mapping function (e.g. Lanyi 1984 and Davis et al 1985). At elevation $=10^{\circ}$, the systematic error in the differenced phase $\Delta \phi$ for $\Delta \theta=1^{\circ}$ is less than $5^{\circ}$ for the dry component but can be as high as $30^{\circ}$ for the wet component. In addition, delay-rate fluctuations are $\sim 1 \mathrm{~cm} / 1-5$ minutes for the wet component and are unknown for the dry component. 
At $5 \mathrm{GHz}$, the total phase-delay contribution of the ionosphere is $\sim 100$ $\mathrm{cm}$ during the day and $\sim 10 \mathrm{~cm}$ during the night with large and unpredictible variations. However, the ionosphere is dispersive and can be removed from the phase-delay if dual frequency observations are conducted (e.g. 2.3 and $8.4 \mathrm{GHz}$ ) and if the program source structure is the same at the two frequencies. This is seldom the case and so difficult monitoring of the ionosphere by Faraday rotation (Gwinn these Proceedings) and by Global Positioning Satellites (GPS) technique (Lanyi and Roth 1988) is required. At $5 \mathrm{GHz}$, systematic error in $\Delta \phi$ for $\Delta \theta=1^{\circ}$ is less than $3^{\circ}$ during the day but as high as $40^{\circ}$ at night at $5 \mathrm{GHz}$. Delay-rate fluctuations are $\sim 7 \mathrm{~cm} / 10-30$ minutes (day) and $\sim 1 \mathrm{~cm} / 10-30$ minutes (night).

Finally, the structure of the reference source must be accounted for in the residual phases $\phi^{R}\left(t_{k}\right)$. The data acquired on the reference source are usually sufficient to make an hybrid map and compute its structure phase with great accuracy (Charlot 1990).

e) Extrapolation of the reference source phase $\phi^{R}\left(t_{k}\right)$ :

As mentioned above, the reference and program sources are often observed alternately. The reference source phase must be extrapolated to the time of observation of the program source, i.e. over 2-6 minutes. Although phase connection is often adopted, other extrapolation scheme can be tried. A useful approach is to use the residual delay-rates of the two reference source scans adjacent to a program source scan at $t_{k}$ and make a double extrapolation. The two phases computed in the forward and reverse senses can be compared at $t_{k}$ and average for $\phi^{R}\left(t_{k}\right)$ in equation (1). The degree of agreement between the two phases is usually $75^{\circ}$ to $100^{\circ}$ when the time interval between the reference and program sources is 3 minutes. As expected, this corresponds to twice the predicted extrapolation phase error $\delta \phi^{R}\left(t_{k}\right)=f \times \delta \dot{\tau} \times\left(t_{k}-t_{k-1}\right)$, i.e. $\delta \phi^{R}\left(t_{k}\right)=36^{\circ}-72^{\circ}$ for the observing frequency $f=5 \mathrm{GHz}$, the residual delay-rate $\delta \dot{\tau}=1-2 \times 10^{-13} \mathrm{sec} / \mathrm{sec}$ due to unmodelled fluctuations of the wet component of the troposphere (Fig. 1) and the time interval between midpoints of consecutive scans $t_{k}-t_{k-1}=180 \mathrm{sec}$. The extrapolation phase error $\delta \phi^{R}\left(t_{k}\right)$ decrease with $t_{k}-t_{k-1}$ and so the source switching cycle duration should be as short as possible. However, statistical tests indicate that the unmodolled delayrate residuals $\delta \dot{\tau}$ are characterized by a Gaussian distribution and, consequently, introduce no biaises in the extrapolated phases over the whole experiment.

\section{IDENTIFICATION OF THE RADIO SOURCE IN}

\section{THE CLOSE BINARY ALGOL}

The result of a series of experiments we conducted to identify the radio emitting region within the close binary Algol is given as an example of the high accuracy differential astrometric measurement that is possible with a very weak radio source. The optically eclipsing close binary Algol is formed of a subgiant $\mathrm{K} 0$ and of a main sequence star B8. The composite spectral type in this binary system makes the identification of the star responsible for the radio emission an interesting astrophysical question. So we designed an astrometric VLBI experiment to measure the displacement of the radio source between the times of the two maximum elongations on the sky. The magnitude of this displacement is 
directly related to the radio emitting star and can be accurately computed from spectroscopic and photometric data. In Algol, this magnitude should be $0.004^{\prime \prime}$ if the radio source is associated with the subgiant $\mathrm{k} 0$ and should be $0.001^{\prime \prime}$ if it is associated with the main sequence star B8 which is 4 times more massive than the subgiant.

We conducted VLBI observations at 4 epochs in April $1989(12,15,16,19)$ scheduled to appropriately sample the orbital phase of Algol and measure twice the positions of the radio emitting region near the two maximum elongations $\Phi_{\text {orb }} \sim 0.25$ and $\Phi_{\text {orb }} \sim 0.75$ ( $\Phi_{\text {orb }}=0.0$ at eclipse). The flux densities of the variable source in Algol were 7, 70, 4 and 6 milliJansky at the four epochs. Phase referencing was used successfully between Algol and the extragalactic source $0309+41\left(\Delta \theta=1^{\circ}\right)$ to coherently add all the visibilities produced by the 3 most sensitive baselines (Phased-VLA with OVRO, Greenbank and Hat Creek) during the 8 hours of each experiment. We analyzed the data as described in section 2 and produce 4 phase-referenced dirty maps similar to the one in Figure 3 . At each of the 4 epochs, we read the position of the map intensity peak relative to the fixed coordinates of the extragalactic source $0309+41$.

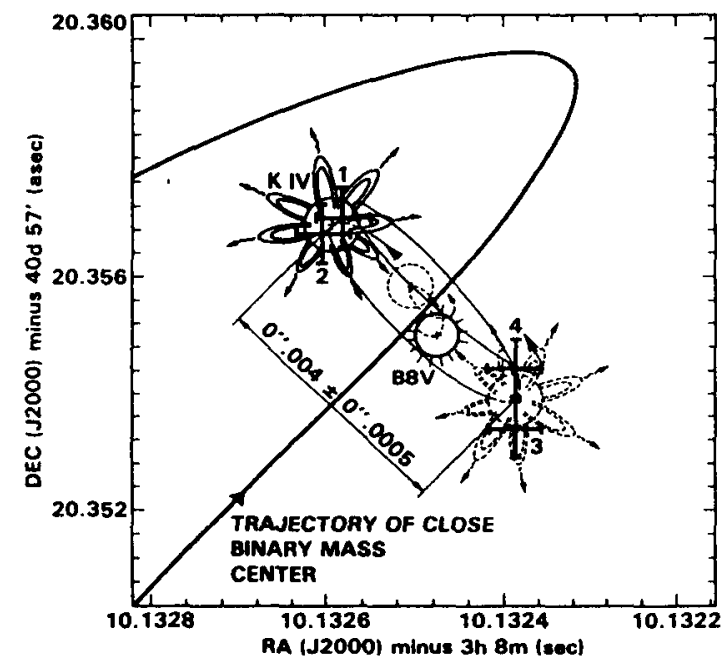
ORBITAL PHASES $\phi$ :
$1: \phi=0.26$
2: $\phi=0.31$
3: $\phi=0.66$
4: $\phi=0.70$

Fig. 4: Positions at 4 epochs (black crosses $1,2,3,4$ ) of the radio source within the close binary Algol between the two maximum sky elongations.

The result is in Figur: 4. The measured displacement between the two eastward $\left(\Phi_{\text {orb }} \sim 0.75\right)$ and the two westward $\left(\Phi_{\text {orb }} \sim 0.25\right)$ positions is $0.004^{\prime \prime} \pm$ $0.0005^{\prime \prime}$. The uncertainty $\left( \pm 0.0005^{\prime \prime}\right)$ is derived from a study of systematic errors in the data analysis. From this displacement, we conclude that the subgiant K0 is the star responsible for the radio emission.

\section{CONCLUSION}

At centimeter wavelength, VLBI phase-referencing can extend the signal coherence time $(T)$ from the typical VLBI scan duration of a few minutes to the 
whole source mutual visibility period of a few hours. This greatly enhances the sensitivity of VLBI (SNR $\propto \sqrt{T}$ ) and allows high-accuracy differential astrometry and imaging of weak radio sources which require the use of the VLBI phase corrected from clock and atmospheric instabilities as the prime observable. We have presented an example of such a differential astrometric result in section 3 and Alef (1988) has been successful in producing a phase-referenced VLBI map of $0248+430$ (0.2 Jy) with Onsala, Bonn, Westerbork, Greenbank, Owens Valley.

Improvements and sophistications of the technique are possible. Various observation strategies could help in calibrating the troposphere and ionosphere without external measurements. Such measurements with water vapor radiometer and satellites are nonetheless promising and it is too early to choose between the two approaches. Reference source phase extrapolation method based on the use of the delay-rate residuals and avoiding the stringent constraints of phase connection should make phase-referencing possible at high frequencies $(22 \mathrm{GHz}$ and $43 \mathrm{GHz}$ ). Especially, if faster switching cycle for alternate observations (total cycle $=1-2$ minutes) is possible to reduce the thermal-like noise in this extrapolation.

\section{ACKNOWLEDGEMENTS}

Haystack Observatory has pioneered the development of phase-referencing in VLBI. The Mark III VLBI result on Algol presented in this contribution would not have been possible withsut crucial help from Drs A.E.E. Rogers and A.R. Whitney. The research described in this report was carried out, in part, by the Jet Propulsion Laboratory, California Institute of Technology, under contract with the National Aeronautics and Space Administration.

\section{REFERENCES}

Alef, W., 1988, IAU Symposium 129, The Impact of VLBI on Astrophysics and Geophysics, ed. M.J. Reid and J.M. Moran, Kluwer, Dordrecht, 523.

Charlot, P. 1990, A. J., 99, 1309.

Davis, J.L., Herring, T.A., Shapiro, I.I., Rogers, A.E.E., Elgered, G., 1985, Rad. Sc., 20, 1593

Gwinn, C., these Proceedings

Lanyi, G.E., 1984, Telecommunication and Data Acquisition Prog. Rep., 42-78, pp. 152-159, Jet Propulsion Laboratory, Pasadena, CA, April-June 1984.

Lanyi, G.E., Roth, T., 1988, Rad. Sc., 23. 483

Lestrade, J-F., Rogers, A.E.E., Whitney, A.R., Niell, A.E., Phillips, R.B., Preston, R.A., 1990, A. J., 99, 1663.

Rogers, A.E.E. et al, 1983, Sciences, 219, 51

Rogers, A.E.E., Moffet, A.T., Backer, D.C., Moran, J.M., 1984, Rad. Sc., 19, 1552.

Sovers, O.J., Fanselow, J.L., 1987, JPL Publication 83-89, Rev. 3, Jet Propulsion Laboratory, Pasadena. CA

Treuhaft, R.N., Lanyi, G.E., 1987 Rad. Sc., 22, 251. 
Goran Pilbratt: Could the use of water vapor radiometry be an alternative to reducing the timescale of switching between the two sources?

J.-F. Lestrade: Absolutely, it is likely to be the only accurate approach at relatively high elevation. The drawback is the present difficulty in operating water vapor radiometers successfully. It is also recognized that they are not providing satisfactory results at low elevation ( $<20$ degrees).

John Conway: What is the maximum target-reference separation that can be used for phase-referencing imaging and what is the limiting factor?

Jean-Francois Lestrade: I have been successful in phase referencing over angular separations ranging from 0.5 to 4.5 degrees.

D. H. Roberts: Your table showed that the ionosphere is a potential problem, even at $5 \mathrm{GHz}$, and certainly at lower frequencies. Can you make use of simultaneous observations in two circular polarizations to remove this effect? J.-F. Lestrade: (No written response was provided) 